\title{
Promoter hypermethylation and reduced expression of RASSF1A are frequent molecular alterations of endometrial carcinoma
}

\author{
Judit Pallarés ${ }^{1}$, Ana Velasco ${ }^{1}$, Nuria Eritja ${ }^{1}$, Maria Santacana ${ }^{1}$, Xavier Dolcet ${ }^{1}$, \\ Miriam Cuatrecasas ${ }^{2}$, Victor Palomar-Asenjo ${ }^{1}$, Lluis Catasús ${ }^{2}$, Jaime Prat $^{2}$ and \\ Xavier Matias-Guiu ${ }^{1}$ \\ ${ }^{1}$ Department of Pathology and Molecular Genetics, Hospital Universitari Arnau de Vilanova, University of \\ Lleida, IRBLLEIDA, Lleida, Spain and ${ }^{2}$ Department of Pathology Hospital de Sant Pau, Barcelona, Spain
}

\begin{abstract}
Alterations in the regulation of the RAS-MAPK pathway are frequent in endometrial carcinoma. RASSF1A is a tumor-suppressor gene that can regulate this pathway negatively. RASSF1A has been found to be inactivated by promoter methylation in some human tumors. The aim of the study was to assess the immunohistochemical expression of RASSF1A in normal endometrium and endometrial carcinoma, and to correlate its expression with K-RAS mutations, presence of microsatellite instability, RASSF1A promoter methylation, and clinicopathological data. RASSF1A immunostaining was evaluated in one tissue microarray constructed from 80 paraffin-embedded samples of normal endometrium, and two tissue microarrays constructed with a total of 157 endometrial carcinomas (one constructed with 95 endometrial carcinomas previously evaluated for $K-R A S$ mutations, and microsatellite instability, and another one containing 62 endometrial carcinomas that were also subjected to RASSF1A promoter methylation analysis). RASSF1A immunostaining was correlated with cell proliferation (Ki67), Cyclin D1 expression and clinicopathological data. Promoter methylation of RASSF1A was assessed by methylation-specific PCR. RASSF1A immunostaining was variable during the menstrual cycle in normal endometrium. RASSF1A expression was significantly reduced in $48 \%$ of endometrial carcinomas, particularly in tumors exhibiting microsatellite instability. RASSF1A-promoter methylation was very frequent in endometrial carcinoma (74\%), and was frequently associated with reduced expression of RASSF1A. RASSF1Apromoter hypermethylation was common in advanced-stage endometrial carcinoma. The results suggest that reduced expression of RASSF1A may play a role in endometrial carcinogenesis by controlling cell proliferation and apoptosis through the MAPK-signaling pathway.
\end{abstract}

Modern Pathology (2008) 21, 691-699; doi:10.1038/modpathol.2008.38; published online 9 May 2008

Keywords: endometrial carcinoma; RASSF1A; tissue microarray; promoter hypermethylation; $K-R A S$; microsatellite instability

Endometrial carcinomas can be classified in two main types; ${ }^{1}$ type I are endometrioid adenocarcinomas. $^{2}$ They usually develop in perimenopausal women and are related to estrogen stimulation. Endometrioid adenocarcinoma may show microsatellite instability ${ }^{3}$ and mutations of the PTEN, ${ }^{4}$ $P I K 3 C A,{ }^{5} K-R A S^{6}$ and $\beta$-catenin genes. ${ }^{7,8}$ Type-II tumors are non-endometrioid carcinomas, which

Correspondence: Dr J Pallarés, MD, PhD, Department of Pathology and Molecular Genetics, Hospital Universitari Arnau de Vilanova, University of Lleida, IRBLLEIDA, Av Alcalde Rovira Roure 80, Lleida 25198, Spain.

E-mail: jpallares@arnau.scs.es

Received 30 August 2007; revised and accepted 23 January 2008; published online 9 May 2008 tend to occur in older women, are unrelated to estrogen stimulation and are frequently associated to p53 mutations and chromosomal instability. ${ }^{9}$

The RAS-signaling pathway plays an important role in tumorigenesis. Mutations in the $R A S$ oncogene have been detected in many different types of tumors. The RAS superfamily of small GTP-binding proteins has a fundamental role in cell growth and differentiation, transcriptional regulation and apoptosis. The frequency of $K-R A S$ mutations in endometrial carcinoma ranges between 10 to $30 \% .{ }^{6}$ In some series, K-RAS mutations have been reported to be more frequent in endometrioid adenocarcinoma showing microsatellite instability. ${ }^{6}$ In these tumors, $K-R A S$ mutations are typically transitions, which may be preceded by abnormal DNA methylation. 
The fact that in endometrioid adenocarcinoma, microsatellite instability frequently coexists with $K-R A S$ methylation-related transitions has lead to the suggestion that these two alterations are close related.

The RASSF1A gene has been recently identified and mapped to chromosome 3p.21.3. RASSF1A is the longest isoform of RASSF1, a new group of RAS effectors thought to regulate cell proliferation and apoptosis. ${ }^{10}$ Expression of RASSF1A has been detected in any normal tissue tested. However, RASSF1A transcripts have been found to be lost in several cancer cell lines. Also, re-expression of $R A S S F 1 A$ dramatically reduces cell growth of tumor cells, supporting a role for $R A S S F 1 A$ as a tumorsuppressor gene. The RASSF1A gene is epigenetically inactivated in a large percentage of different human malignancies, including cancers of the lung, breast, kidney, bladder, stomach, ovary and neuroblastoma. ${ }^{11}$

RASSF1A can either associate with oncogenic/ mutant K-RAS or with GTP-bound K-RAS through heterodimerization with NORE1, a RAS-GTP-binding protein. ${ }^{12}$ This assumption raises the hypothesis of $K-R A S-B R A F-R A S S F 1 A$ genes as partners in the same pathway.

Promoter methylation is a frequent mechanism of gene inactivation of endometrioid adenocarcinoma. It may involve many different genes participating in cell signalling, apoptosis and cell proliferation ( $p 14$, PTEN, p16, TSP-1, IGF-2, estrogen receptor- $\alpha$, HIC-1, $M L H-1)$. Inactivation of $h M H L-1$ by promoter hypermethylation is present in the majority of endometrioid adenocarcinoma with microsatellite instability, which suggests that abnormal methylation is the first step in the acquisition of the mutator phenotype in endometrial carcinogenesis. ${ }^{13}$ Since altered methylation status is frequent in endometrioid adenocarcinoma with microsatellite instability, and also frequently coexists with K-RAS mutations, we may hypothesize that RASSF1A promoter hypermethylation could be frequent in endometrial carcinoma, possibly coexisting with any of these phenomena.

The main aim of the present study was to assess the frequency of reduced expression of RASSF1A and $R A S S F 1 A$-promoter hypermethylation in endometrial carcinoma in correlation with the presence of microsatellite instability, K-RAS mutations and clinicopathological data. Also, the purpose of this study was to assess immunohistochemical expression of RASSF1A in normal endometrium, in different phases of the menstrual cycle, using microarray technology.

\section{Materials and methods}

\section{Tissue Microarrays}

Three tissue microarrays were constructed. The $f$ irst tissue microarray was constructed from 80 paraffin-embedded samples of normal endometrium in different phases of the menstrual cycle, obtained from the surgical pathology files from Hospital Universitari Arnau de Vilanova de Lleida. The second tissue microarray was composed of 95 endometrial carcinomas, previously evaluated for $K-R A S$ mutations and microsatellite instability. ${ }^{3,6}$ The third tissue microarray was constructed from 62 endometrial carcinomas that were also subjected to RASSF1A-promoter methylation (see below). Overall, the two last tissue microarrays comprised 157 endometrial carcinomas, obtained from the surgical pathology files of Hospital Universitari Arnau de Vilanova de Lleida and Hospital de Sant $\mathrm{Pau}$, Barcelona, Spain. They included 47 grade-I endometrioid adenocarcinomas, 58 grade-II endometrioid adenocarcinomas, 26 grade III endometrioid adenocarcinomas, 15 serous carcinomas, four clear-cell carcinomas and seven mixed Müllerian malignant tumors. One hundred and eight tumors were stage-I, 15 stage-II carcinoma, 22 stage-III and one was a stage-IV carcinoma. Staging information was incomplete for 11 cases. The study was approved by the local ethical committee. Specific informed consent was obtained from all subjects.

A Tissue Arrayer device (Beecher Instrument, MD, USA) was used to construct tissue microarrays. Briefly, all the samples were histologically reviewed and representative areas were marked in the corresponding paraffin blocks. Two selected cylinders (0.6 $\mathrm{mm}$ in largest diameter) from two different areas were included in each case. Control normal tissues from the same endometrial carcinoma specimens were also included.

\section{Immunohistochemical Study}

Tissue-microarray blocks were sectioned at a thickness of $3 \mu \mathrm{m}$, dried for $16 \mathrm{~h}$ at $56^{\circ}$ before being de-waxed in xylene and rehydrated through a series of graded ethanol concentrations, followed by wash with phosphate-buffered saline. Antigen retrieval was achieved by heat treatment in a pressure cooker for 2 min in EDTA ( $\mathrm{pH}$ 8.9). Before staining sections, endogenous peroxidase activity was blocked. The antibodies used were as follows: anti-RASSF1A (1:500 dilution; eB114-20H1; eBioscience), Ki67 (1:100 dilution; MIB-1; Dako) and cyclin D1 (1:25 dilution; DCS6; Dako). After incubation, the reaction was visualized with the EnVision Detection kit (Dako), using diaminobenzidine chromogen as a substrate. Sections were counterstained with hematoxylin. Appropriate positive and negative controls were also tested.

Immunohistochemical results were evaluated by two pathologists following uniform pre-established criteria. RASSF1A immunoexpression was graded semi-quantitatively by considering the percentage and intensity of the staining. A histological score 
was obtained from each sample, which ranged from 0 (no immunoreaction) to 300 (maximum immunoreactivity). The score was obtained by applying the following formula: Histoscore $=1 \times(\%$ light staining $)+2 \times(\%$ moderate staining $)+3 \times(\%$ strong staining). The reliability of such score for interpretation of immunohistochemical staining in endometrial carcinoma tissue microarrays has been proven previously. ${ }^{14-17}$ Since each tissue microarray included two different tumor cylinders from each case, immunohistochemical evaluation was conducted after examining both samples. Finally, the percentage of positive nuclei in each case was used to assess the cellular proliferation (Ki67) and the cyclin D1 expression.

The reproducibility of tissue-microarray immunostaining was confirmed by comparing results from tissue microarray with those obtained in sections from the corresponding paraffin blocks of 37 randomly selected cases. The overall concordance was $89.2 \%$. The Kappa index of agreement between the two methods ranged from 0.68 to 0.83 .

\section{Methylation-Specific PCR}

Genomic DNA was isolated with the DNAeasy tissue kit (Qiagen GmbH, Hilden, Germany), from normal and tumor tissue from 57 patients with endometrial carcinoma. Samples were frozen after surgery and corresponded to 57 of the 62 endometrial carcinoma samples of the third tissue microarray. A specific informed consent was used and the study was approved by the local ethical committee. DNA was subjected to bisulfite treatment. Briefly, $1 \mu \mathrm{g}$ of DNA was denatured by sodium hydroxide and modified by sodium bisulfite. DNA samples were then purified and treated again with sodium hydroxide, precipitated with ethanol and resuspended in water (EZ DNA Methylation kit; ZYMO RESEARCH, USA). RASSF1A-promoter methylation status was determined following protocols from previous studies. ${ }^{18}$ DNA was amplified with primers MF (GTGTTAACGCGTTGCGTATC) and MR (AACCCCG CGAACTGAAAAACGA) for methylated DNA and UF (TTTGGTTGGAGTGTGTTAATGTG) and UR (CAAACCCCACAAACTAAAACAA) for unmethylated DNA. Each PCR reaction contained $1 \times$ buffer, $1.5 \mathrm{mM} \mathrm{MgCl}_{2}$ and $1 \mathrm{U} / \mu \mathrm{l}$ TaqGold (Applied Biosystems Inc., Santa Clara, CA, USA); $0.2 \mathrm{mM}$ dNTPs (Biotools B\&M Labs, SA, Madrid, Spain) and $0.2 \mu \mathrm{M}$ of each primer. PCR conditions were as follows: an initial 10 -min denaturation at $96^{\circ} \mathrm{C}$ followed by two cycles of $30 \mathrm{~s}$ at $95^{\circ} \mathrm{C}, 30 \mathrm{~s}$ at $67^{\circ} \mathrm{C}, 30 \mathrm{~s}$ at $72^{\circ} \mathrm{C}$; an additional two cycles of $30 \mathrm{~s}$ at $95^{\circ} \mathrm{C}, 30 \mathrm{~s}$ at $65^{\circ} \mathrm{C}, 30 \mathrm{~s}$ at $72^{\circ} \mathrm{C}$, a further two cycles of $30 \mathrm{~s}$ at $95^{\circ} \mathrm{C}, 30 \mathrm{~s}$ at $63^{\circ} \mathrm{C}, 30 \mathrm{~s}$ at $72^{\circ} \mathrm{C}$ cycles and a final extension step of $10 \mathrm{~min}$ at $72^{\circ} \mathrm{C}$. Each PCR product was directly loaded on $3 \%$ agarose gel, stained with ethidium bromide and visualized under UV illumination.

\section{Statistical Analysis}

Statistical analysis was performed with a database using SPSS for Windows (version 11.5; SPSS Inc., Chicago, IL, USA). Immunohistochemical results were compared by Mann-Whitney $U$ and Student's t-test when applicable. Associations between qualitative variables were assessed by $\chi^{2}$ - or Fisher's exact tests. Correlations between quantitative variables were established through Pearson and Spearman $\rho$ tests. Statistical significance was set at $P \leq 0.05$.

\section{Results}

\section{Immunohistochemistry}

In normal endometrium, RASSF1A was evaluated in all the 80 cases that were included in the first tissue microarray. RASSF1A immunostaining varied according to the different phases of the menstrual cycle. RASSF1A immunoexpression was significantly higher in the proliferative phase (mean Histoscore, 45.96) than in the secretory endometrium (mean Histoscore, 31.58) $(P=0.001)$ (Figure 1). Staining was cytoplasmic. Stromal cells were positive in both, the proliferative and the secretory phases.

In endometrial carcinoma, RASSF1A was evaluated in 148 out of the 157 cases that were included in the second and third tissue microarrays. Six cases were excluded as there was no representative tumor tissue in the tissue microarray sections, and three cases were missed in the construction of tissue microarray. Overall, RASSF1A immunoexpression was significantly reduced in endometrial carcinoma as compared with normal endometrium. Mean RASSF1A Histoscore was 38.28 in endometrial carcinoma and 70.00 in normal endometrium $(P=0.007)$. We observed negative staining for RASSF1A in 71 cases of endometrial carcinoma (48\%), whereas remaining 77 cases were positive $(52 \%)$. Staining was cytoplasmic and heterogeneous, and Histoscore ranged from 10 to 290 (Figures 2 and 3). Nuclear immunostaining was observed in 11 cases.

A slightly reduced RASSF1A immunostaining was observed in non-endometrioid endometrial carcinomas (mean Histoscore, 25.25) as compared with endometrioid endometrial carcinoma (mean Histoscore, 30.18). However, these differences were not statistically significant $(P=0.457)$. The correlation between RASSF1A immunoexpression and histological grade or pathological stage did not get any significant difference $(P=0.771$ and $P=0.303$, respectively). There was no significant correlation between cell proliferation (Ki67) and RASSF1A immunoexpression $(r=0.088, P=0.446)$. Cyclin D1 nuclear expression was observed in $64.79 \%$ cases of endometrial carcinoma, and no correlation was found with RASSF1A protein expression $(P=0.635)$. 

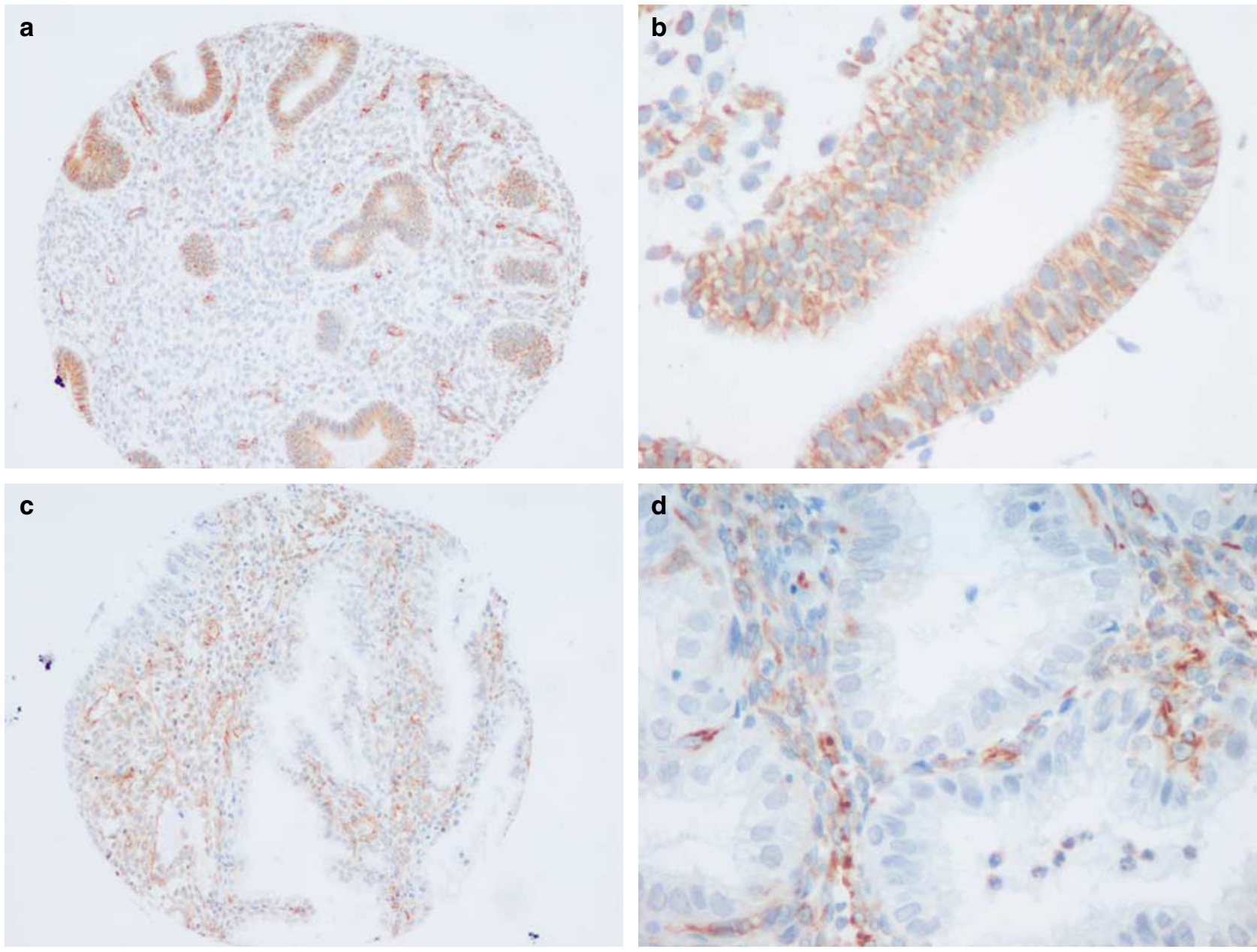

Figure 1 RASSF1A immunostaining was higher in the proliferative phase (a, b) than in the secretory phase (c, d).
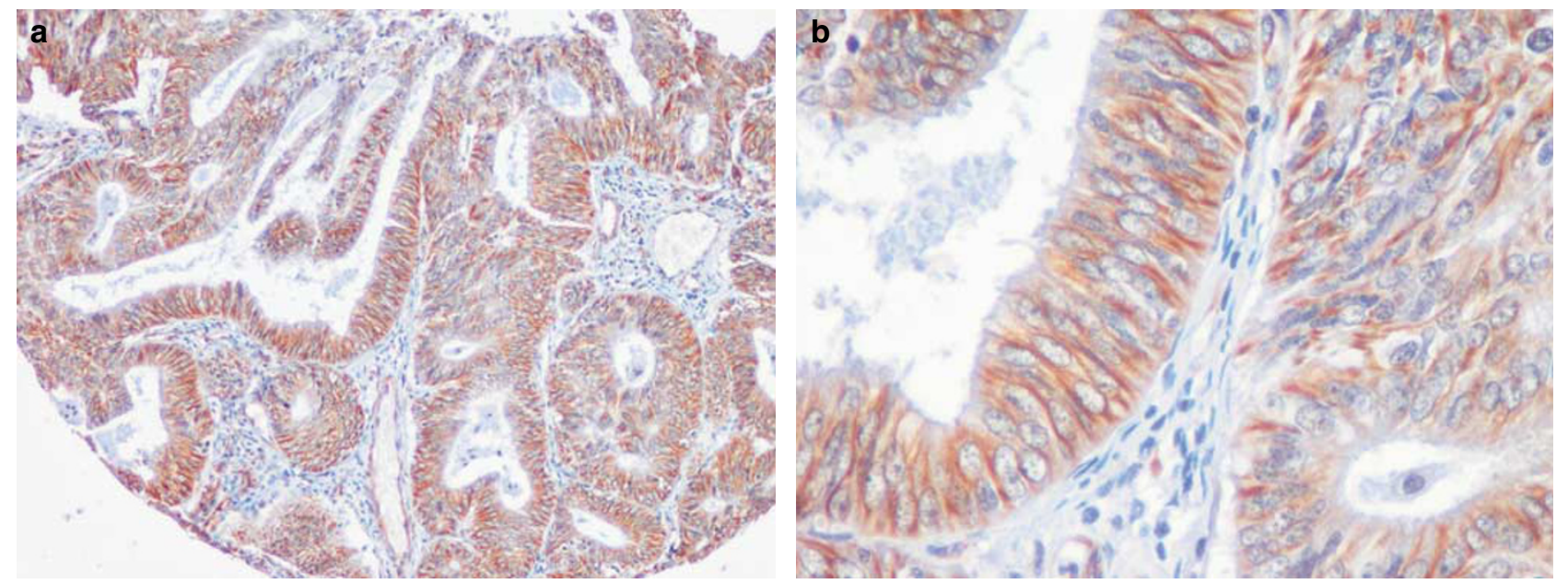

Figure 2 RASSF1A immunoexpression in an endometrial carcinoma without RASSF1A-promoter hypermethylation. Notice the strong cytoplasmic staining $(\mathbf{a}, \mathbf{b})$.

Finally, RASSF1A immunoexpression was correlated with microsatellite instability and $K-R A S$ mutations in 67 cases of endometrial carcinoma from the second tissue microarray. No significant correlation was found when we considered total absence of RASSF1A expression (Histoscore 0) as 

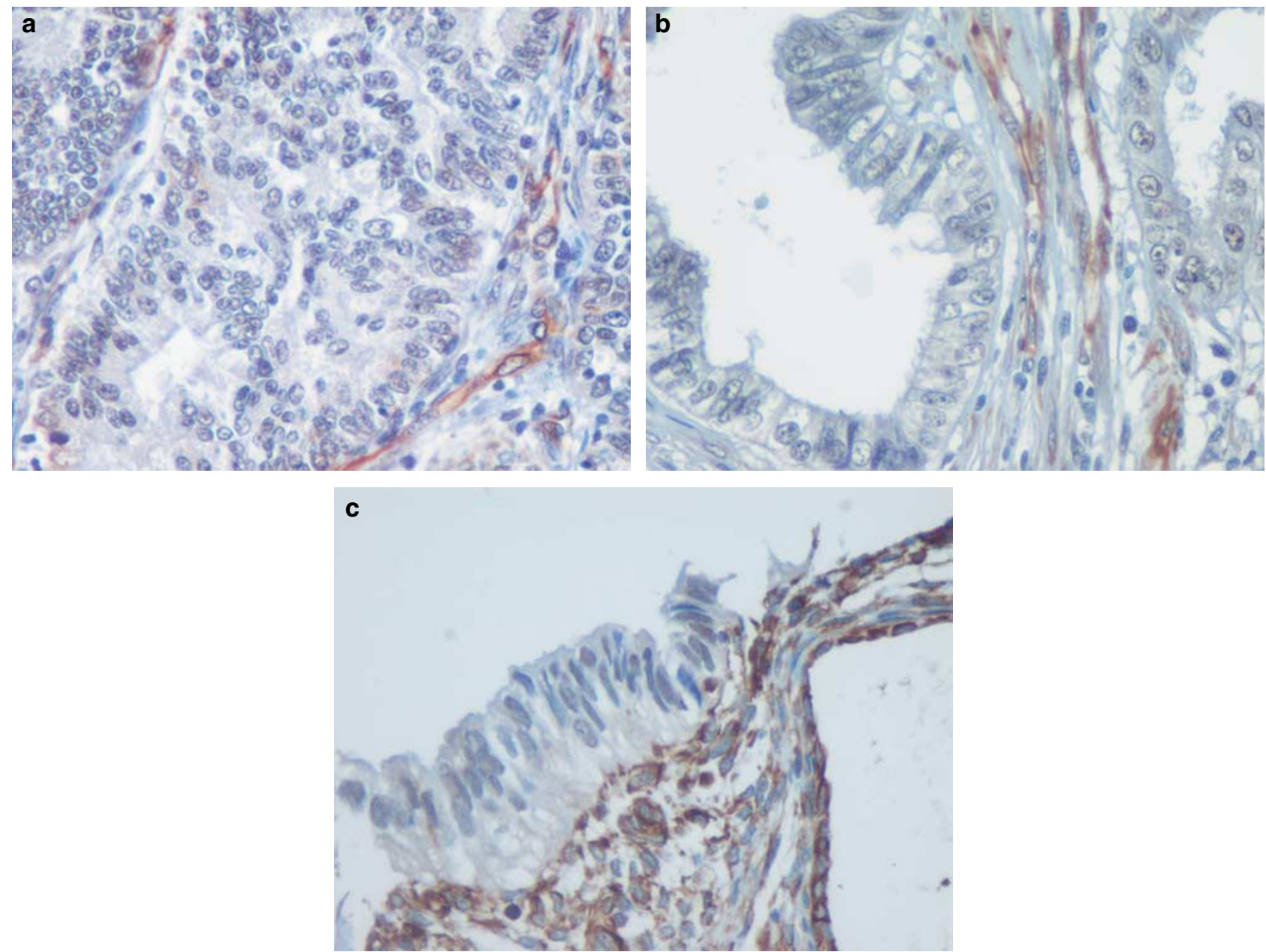

Figure 3 RASSF1A immunostaining in an endometrial carcinoma with RASSF1A-promoter hypermethylation. Notice that the cytoplasmic staining $(\mathbf{a}, \mathbf{b})$ is lower than in the case illustrated in Figure 2a and b. The corresponding normal tissue, exhibited positive staining (c).

negative $(P=0.510$ and $P=0.294)$. However, association between RASSF1A immunostaining and presence of microsatellite instability turned out to be significant when we considered as negative those endometrial carcinoma with a Histocore less than 50. In other words, 18 of the 20 endometrial carcinomas with microsatellite instability exhibited Histoscores less than $50(P=0.042)$ (Table 1). Moreover, seven from the 10 cases with $K-R A S$ mutations were negative for RASSF1A immunoexpression (Histoscore less than 50), but differences were not statistically significant $(P=0.540)$ (Table 1$)$.

\section{RASSF1A-Promoter Hypermethylation}

RASSF1A-promoter hypermethylation was detected in 42 of the $57(74 \%)$ endometrial carcinoma samples that were tested (Figure 4). In some cases, the degree of promoter methylation varied in different areas of the tumor (Figure 4, case 2, T1 and T2). Promoter methylation was not detected in normal tissue samples from the same patients with
Table 1 RASSF1A immunoexpression according to microsatellite instability and $K-R A S$ mutational status

\begin{tabular}{lccccc}
\hline & \multicolumn{2}{c}{ Microsatellite instability } & & \multicolumn{2}{c}{ K-RAS mutations } \\
\cline { 2 - 3 } \cline { 5 - 6 } & Positive & Negative & & Positive & Negative \\
\hline RASSF1A positive & 2 & 16 & & 3 & 15 \\
$\begin{array}{l}\text { RASSF1A negative } \\
\text { (Hscore <50) }\end{array}$ & 18 & 31 & & 7 & 42 \\
Total & 20 & 47 & & 10 & 57 \\
\hline
\end{tabular}

endometrial carcinoma. Interestingly, the frequency of RASSF1A-promoter methylation increased in correlation with pathological stage (Table 2). RASS$F 1 A$-promoter methylation was observed in $67 \%$ of stage-IA or stage-IB endometrial carcinoma cases $(18 / 26)$ as compared with $80 \%$ of stage-IC endometrial carcinomas (10/14) and $100 \%$ of stage-IIIA endometrial carcinomas (6/6). The vast majority of RASSF1A unmethylated tumors were stage-IA endometrial carcinomas $(54.5 \%)(6 / 11)$, and only two 


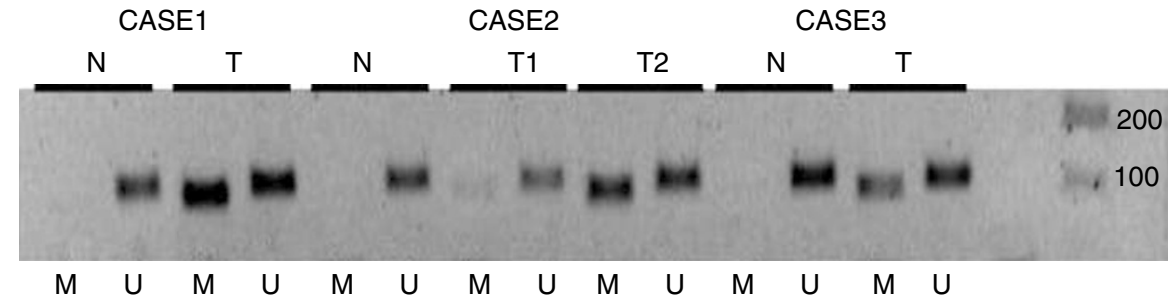

Figure 4 MS-PCR for the RASSF1A gene in endometrial carcinoma and normal tissue. U, PCR product with primers specific for unmethylated DNA; M, PCR product with primers specific for methylated DNA. RASSF1A is methylated in the three cases of endometrial carcinoma. Notice that the degree of promoter hypermethylation differed between two different areas of one tumor (T1 and T2) of case 2.

Table 2 RASSF1A methylation status according to pathological stage

\begin{tabular}{lccr}
\hline Stage & $\begin{array}{c}\text { RASSF1A } \\
\text { methylated }\end{array}$ & $\begin{array}{c}\text { RASSF1A } \\
\text { unmethylated }\end{array}$ & Total \\
\hline IA & 5 & 6 & 11 \\
IB & 13 & 3 & 16 \\
IC & 10 & 4 & 14 \\
IIA & 2 & 0 & 2 \\
IIB & 6 & 2 & 8 \\
IIIA & 6 & 0 & 6
\end{tabular}

of the 16 stage-II or stage-III endometrial carcinomas were unmethylated (12\%). RASSF1A-promoter hypermethylation was more frequent among endometrioid carcinomas (75\%) (37 of 49 cases) as compared with non-endometrioid carcinomas $(62.5 \%)$ (5 of 8 cases), but these differences were not statistically significant $(P=0.422) . R A S S F 1 A$ was also analyzed in correlation with histological grade. Grade-I and grade-II endometrial carcinomas were more frequently methylated $(71$ and $86 \%$, respectively) than grade-III tumors (57\%). However, the differences were not significant $(P=0.152)$.

\section{Correlation between RASSF1A Immunostaining and RASSF1A-Promoter Hypermethylation}

Finally, we assessed the correlation between RASSF1A immunoexpression and the methylation status of the promoter region of the gene in 53 of the 57 initial cases studied for $R A S S F 1 A$-promoter hypermethylation. RASSF1A was methylated in 26 of 27 cases negative for RASSF1A immunostaining (Table 3). As expected, RASSF1A protein expression was dramatically reduced in cases with $R A S S F 1 A$ promoter methylation (mean Histoscore, 30.38) as compared with unmethylated cases (mean: 101.92), and differences were statistically significant $(P=0.000)$. However, 14 cases exhibited RASSF1A immunostaining in the presence of RASSF1Apromoter methylation. Interestingly, RASSF1A staining in these cases was heterogeneous and weaker. A comparison between cases with positive or negative RASSF1A-promoter hypermethylation among endometrial carcinoma with
Table 3 Correlation between methylation and RASSF1A immunoexpression

\begin{tabular}{cccc}
\hline & $\begin{array}{c}\text { RASSF1A } \\
\text { methylated }\end{array}$ & $\begin{array}{c}\text { RASSF1A } \\
\text { unmethylated }\end{array}$ & Total \\
\hline $\begin{array}{c}\text { RASSF1A-positive } \\
\text { immunostaining }\end{array}$ & 14 & 12 & 26 \\
$\begin{array}{c}\text { RASSF1A-negative } \\
\text { immunostaining }\end{array}$ & 26 & 1 & 27 \\
\hline
\end{tabular}

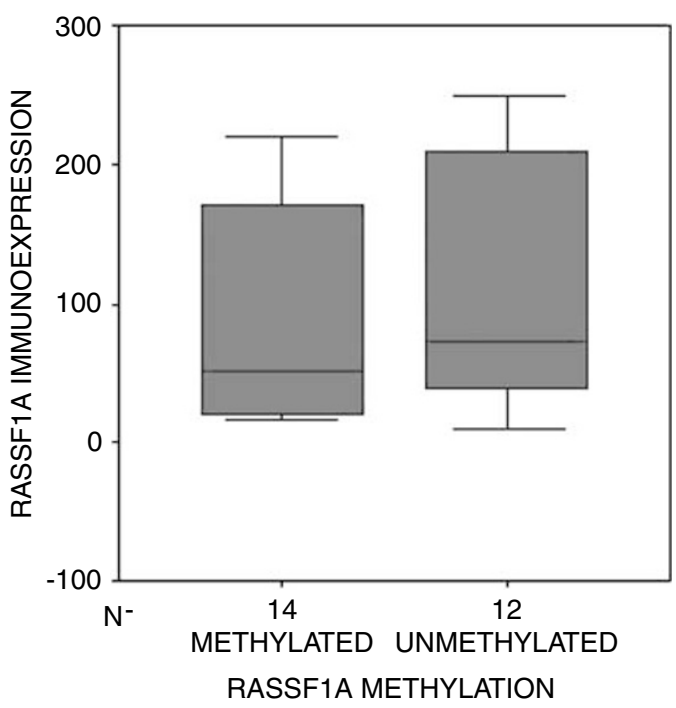

Figure 5 Box-plot comparison of RASSF1A immunoexpression and methylation status.

positive RASSF1A staining was performed. Interestingly, endometrial carcinoma cases with RASSF1Apromoter methylation showed reduced RASSF1A immunoexpression (mean Histoscore, 86.09) as compared with unmethylated cases (mean Histoscore, 110.42) (Figure 5), but these differences were not significant $(P=0.274)$.

\section{Discussion}

Epigenetic inactivation is defined as a change imposed onto the functionality of a gene that does not involve an alteration of its coding sequence. Transcriptional silencing by hypermethylation of 
CpG islands in the promoter regions of tumorsuppressor genes is becoming a common phenomenon in carcinogenesis. Although this phenomenon is seen in a wide spectrum of tumors, it is a frequent mechanism of tumor-suppressor gene inactivation in endometrial carcinoma. De novo methylation of the RASSF1A promoter is one of the most frequent inactivation events detected in human cancer and leads to silencing of RASSF1A expression. ${ }^{11}$ The C-terminus of RASSF1A is homologous to the mammalian RAS-effector protein NORE1 and encodes a RAS-association domain. Three major splice variants, RASSF1A, RASSF1B and RASSF1C, are transcribed from two different CpG islands, separated approximately by $3.5 \mathrm{~kb} .{ }^{19}$

In the present study, we have assessed the immunohistochemical expression and the presence of promoter hypermethylation of the RASSF1A gene in a series of endometrial carcinomas, and detected reduced expression of RASSF1A in $48 \%$ of the cases and promoter hypermethylation in $73 \%$. Moreover, there was a statistically significant association between both the phenomena. Although RASSF1Apromoter hypermethylation has previously been evaluated in endometrial carcinoma, this is the first study in the literature to demonstrates that it is associated with reduced RASSF1A protein expression, in a large series of endometrial carcinoma and a tissue-microarray approach. In other studies, the frequency of $R A S S F 1 A$-promoter hypermethylation in endometrial carcinoma has been reported to be between 30 and $85 \%$, as found by methylationspecific PCR (MS-PCR). ${ }^{20,21}$ Moreover, in a recent study Kang et al found promoter hypermethylation in two of four endometrial carcinoma cell lines tested for promoter hypermethylation (AN3-CA, HEC-1-B, KLE, RL95-2). They also demonstrated that endometrial carcinoma cell lines with promoter hypermethylation, treated with 5-aza-2-deoxycytidine, a drug that inhibits DNA methylation, restored RASSF1A expression.

RASSF1A protein expression was heterogeneous in our endometrial carcinoma samples. Some cases were RASSF1A positive in many areas and negative in some foci, and also some RASSF1A-negative samples contained a small proportion of cells showing strong staining. In our opinion, the heterogeneous distribution of RASSF1A-promoter hypermethylation is the reason that explains why we detected RASSF1A-promoter hypermethylation in tumors with positive immunostaining for RASSF1A. This phenomenon can also be found in other types of tumors and with other genes that are silenced by promoter methylation. For example, it has recently been seen that a heterogeneous protein expression of some genes, including RASSF1A, is seen in about $70 \%$ of malignant melanoma cases, due to intra-tumoral distribution of promoter hypermethylation. ${ }^{22}$

During tumorigenesis, activated $R A S$ is usually associated with enhanced proliferation, transformation and cell survival. RAS effectors, like RASSF1A, are supposed to have an inhibitory growth signal, which needs to be inactivated during tumorigenesis. Contradictory results between RASSF1A inactivation and $K-R A S$ mutation have been obtained using different types of tumors. They were mutually exclusive events in colorectal and pancreatic cancer, ${ }^{23,24}$ but the correlation was not significant in lung cancer. ${ }^{25}$ Finally, in thyroid cancer, RASSF $1 A$ methylation is mutually exclusive with mutations in $B R A F{ }^{26}$ Since $B R A F$ mutations are very infrequent in endometrial carcinoma, we focused our interest in the correlation between $R A S S F 1 A$ inactivation and $K-R A S$ mutations and microsatellite instability.

In our series of endometrial carcinoma, seven out of 10 cases with $K-R A S$ mutations were negative for RASSF1A immunoexpression. Kang et al found RASSF1A hypermethylation to be associated very strongly with microsatellite instability, and inversely correlated with $K-R A S / B R A F$ mutations in microsatellite instability-negative endometrial carcinomas. Very recently, Pijnenborg et al reported RASSF1A hypermethylation in $85 \%$ of recurrent endometrial carcinoma, without finding any correlation between RASSF1A-methylation status and $K-R A S$ or BRAF mutations. Interestingly, they demonstrated hypermethylation of RASSF $1 A$ in samples of endometrial hyperplasias $(50 \%)$, cyclic $(21 \%)$ and atrophic endometrium (38\%). The authors suggest a role of $R A S S F 1 A$ methylation as an early event in endometrial carcinogenesis. However, we have not been able to detect RASSF1Apromoter hypermethylation in any of the normal tissue samples corresponding to the 57 cases of endometrial carcinoma, Moreover, the staining pattern that we obtained in normal endometrial tissue was dependent on the menstrual cycle phase, which suggests that in normal endometrium, RASS$F 1 A$ is under the regulation of steroid hormonesignaling pathways.

Microsatellite instability occurs in $25-30 \%$ of sporadic endometrial carcinoma, and is characteristic of endometrioid adenocarcinoma. The identification of CpG-island methylation in several genes in colonic and gastric carcinomas with microsatellite instability suggested a relationship between altered methylation and the microsatellite-mutator phenotype. In the present study, we analyzed the possible association between RASSF1A expression and the microsatellite instability status in our tissue-microarray approach. We observed a statistically significant association between decreased RASSF1A protein expression and microsatellite instability when we considered as negative those tumors exhibiting RASSF1A Histoscore of less than 50. Our results support the data of Kang and co-workers who also demonstrated that RASSF1A methylation was strongly associated with microsatellite instability and $h M L H-1$ methylation. Similar results were also obtained in colorectal cancer in which a higher frequency of RASSF1A-promoter methylation has 
been described in microsatellite instability + colorectal cancers as compared with tumors without the microsatellite instability phenotype. The results suggest that the methylation affecting RASSF1A promoter may preferentially occur within the context of microsatellite instability + endometrioid adenocarcinoma.

In the present series, we have observed a higher frequency of RASSF1A-promoter methylation in correlation with advanced pathological stage and poorly differentiated carcinomas. In agreement with this result, Jo et al, ${ }^{27}$ found that $R A S S F 1 A$ methylation was significantly more frequent in endometrial carcinoma in association with advanced stage (III, IV), lymph node involvement and high histological grade. Moreover, they demonstrated higher incidence of recurrences and lower disease-free survival in patients with $R A S S F 1 A$ hypermethylation.

Finally, we noted a nuclear immunostaining of RASSF1A in some cases of endometrial carcinoma. Biochemical studies have demonstrated that RASSF1A is a microtubule-binding protein, which regulates mitotic progression. ${ }^{28}$ It has also been shown that RASSF1A overexpression induces mitotic arrest at the metaphase with aberrant mitotic cells. ${ }^{28}$ RASSF1A regulates mitosis by inhibiting the anaphase-promoting complex through Cdc20, and induces G2-M arrest at the prometaphase. Also, RASSF1A prevents degradation of cyclin D1 and delays mitotic progression. ${ }^{29}$ RASSF1A is also able to affect other cyclins, such as cyclin D3 or cyclin A, through interaction with transcription factor $\mathrm{p} 120^{\mathrm{E} 4 \mathrm{~F}}$, which induces cell-cycle arrest and provides a mechanistic link with other known tumorsuppressor genes such as $p 14^{A R F}, R b$ and $p 53$ that are known to interact with $\mathrm{p} 120^{\mathrm{E} 4 \mathrm{~F}} \cdot{ }^{30}$ To evaluate the possible relationship between RASSF1A and the cell-cycle machinery, we checked the relation between RASSF1A expression with cyclin D1 expression or proliferation index (Ki67 staining), but found no significant association.

In summary, we report, for the first time, reduced protein expression of RASSF1A in endometrial carcinoma using tissue-microarray technology, and reduced RASSF1A immunoexpression in cases with promoter hypermethylation. Also, we demonstrate higher frequency of $R A S S F 1 A$-promoter methylation in advanced endometrial carcinoma stage, suggesting that $R A S S F 1 A$ could be a useful indicator of tumor aggressiveness in endometrial carcinoma patients.

\section{Acknowledgements}

This work was supported by grants FIS PI060577, FIS PI070276, SAF2002-10529-E, SAF2004-05250; Marató de TV3 2005-47 and programa de intensificación de la investigación, Instituto Carlos III. XD holds a postdoctoral fellowship from Fondo de
Investigaciones Sanitarias, Ministerio de Sanidad y Consumo (CP05/00028).

\section{Conflict of interest}

The authors state no conflict of interest.

\section{References}

1 Bockman JV. Two pathogenetic types of endometrial carcinoma. Gynecol Oncol 1983;15:10-17.

2 Matias-Guiu X, Catasus L, Bussaglia E, et al. Molecular Pathology of endometrial hyperplasia and carcinoma. Hum Pathol 2001;32:569-577.

3 Catasús L, Machín P, Matias-Guiu X, et al. Microsatellite instability in endometrial carcinomas. Clinicopathologic correlations in a series of 42 cases. Hum Pathol 1998;29:1160-1164.

4 Bussaglia E, del Rio E, Matias-Guiu X, et al. PTEN mutations in endometrial carcinomas. A molecular and clinicopathologic analysis of 38 cases. Hum Pathol 2000;31:312-317.

5 Velasco A, Bussaglia E, Pallares J, et al. PIK3CA gene mutations in endometrial carcinoma. Hum Pathol 2006;37:1465-1472.

6 Lagarda H, Catasus L, Argüelles RM, et al. k-RAS mutations in endometrial carcinomas with microsatellite instability. J Pathol 2001;193:193-199.

7 Machin P, Catasus L, Pons C, et al. CTNNB1 mutations and beta-catenin expression in endometrial carcinomas. Hum Pathol 2002;33:206-212.

8 Moreno-Bueno G, Hardisson D, Prat J, et al. Abnormalities of the APC/beta-catenin pathway in endometrial cancer. Oncogene 2002;21:7981-7990.

9 Prat J, Oliva E, Lerma E, et al. Uterine papillary serous adenocarcinoma. A 10 case study of p53 and c-erbB 2 expression and DNA content. Cancer 1994;74: 1778-1783.

10 Dammann R, Schagdarsurengin U, Strunnikova M, et al. Epigenetic inactivation of the Ras-association domain family 1 (RASSF1A) gene and its function in human carcinogenesis. Histol Histopathol 2003;18: 665-677.

11 Pfeiffer GP, Dammann R. Methylation of the tumor suppressor gene RASSF1A in human tumors. Biochem 2005;70:576-583.

12 Ortiz-Vega S, Khokhlatchev A, Nedwidek M, et al. The putative tumor suppressor RASSF1A homodimerizes and heterodimerizes with the Ras-GTP binding protein Nore1. Oncogene 2002;21:1381-1390.

13 Esteller M, Catasus L, Matias-Guiu X, et al. hMLH1 promoter hypermethylation is an early event in human endometrial tumorigenesis. Am J Pathol 1999;155: 1767-1772.

14 Pallares J, Martínez-Guitarte JL, Dolcet X, et al. Abnormalities in NF- $\kappa \mathrm{B}$ family and related proteins in endometrial carcinoma. J Pathol 2004;204: 569-577.

15 Pallares J, Martínez-Guitarte JL, Dolcet X, et al. Survivin expression in endometrial carcinoma. Int J Gynecol Pathol 2005;24:247-253.

16 Pallares J, Martínez-Guitarte JL, Dolcet X, et al. Immunohistochemical analysis of PTEN in endometrial carcinoma. Mod Pathol 2005;18:719-727. 
17 Dolcet X, Llobet D, Pallares J, et al. FLIP is frequently expressed in endometrial carcinoma and has a role in resistance to TRAIL-induced apoptosis. Lab Invest 2005;85:885-894.

18 Nakamura N, Carney JA, Jin L, et al. RASSF1A and NORE1A methylaton and $B R A F^{\mathrm{V} 600 \mathrm{E}}$ mutations in thyroid tumors. Lab Invest 2005;85:1065-1075.

19 Dammann R, Li C, Yoon JH, et al. Epigenetic inactivation of a Ras-association domain family protein from the lung tumour suppressor locus 3p21.3. Nat Genet 2000;25:315-319.

20 Kang S, Lee JM, Jeon ES, et al. RASSF1A hypermethylation and its inverse correlation with BRAF and/or KRAS mutations in MSI-associated endometrial carcinoma. Int J Cancer 2006;119:1316-1321.

21 Pijnenborg JMA, Dam-de Veen GC, Kisters N, et al. RASSF1A methylation and K-ras and B-raf mutations and recurrent endometrial cancer. Ann Oncol 2007;18:491-497.

22 Rastetter M, Schagdarsurengin U, Lahtz C, et al. Frequent intra-tumoral heterogeneity of promoter hypermethylation in malignant melanoma. Histol Histopathol 2007;22:1005-1015.

23 Van Engeland M, Roemen GM, Brink M, et al. K-ras mutations and RASSF1A promoter methylation in colorectal cancer. Oncogene 2002;21:3792-3795.
24 Dammann R, Schagdarsurengin U, Liu L, et al. Frequent RASSF1A promoter hypermethylation and K-ras mutations in pancreatic carcinoma. Oncogene 2003;22:3806-3812.

25 Kim DH, Kim JS, Park JH, et al. Relationship of Ras association domain family 1 methylation and K-ras mutation in primary non-small cell lung cancer. Cancer Res 2003;63:6206-6211.

26 Xing M, Cohen Y, Mambo E, et al. Early occurrence of RASSF1A hypermethylation and its mutual exclusion with BRAF mutation in thyroid tumorigenesis. Cancer Res 2004;64:1664-1668.

27 Jo H, Kim JW, Kang GH, et al. Association of promoter hypermethylation of the RASSF1A gene with prognostic parameters in endometrial cancer. Oncol Res 2006;16:205-209.

28 Liu L, Tommasi S, Lee DH, et al. Control of microtubule stability by the RASSF1A tumor suppressor. Oncogene 2003;22:8125-8136.

29 Song MS, Song SJ, Ayad NG, et al. The tumor suppressor RASSF1A regulates mitosis by inhibiting the APC-Cdc20 complex. Nat Cell Biol 2004;6: 129-137.

30 Fajas L, Paul C, Vié A, et al. Cyclin A is a mediator of p120E4F-dependent cell cycle arrest in G1. Mol Cell Biol 2001;21:2956-2966. 